\title{
Germanica
}

\section{Cette beauté qui tue. Le beau et le mythe des sirènes}

\section{Françoise Rétif}

\section{Q OpenEdition}

\section{Journals}

Édition électronique

URL : http://journals.openedition.org/germanica/453

DOI : 10.4000/germanica.453

ISSN : 2107-0784

Éditeur

Université de Lille

Édition imprimée

Date de publication : 1 décembre 2005

Pagination : 79-87

ISBN : 2-913857-16-7

ISSN : 0984-2632

Référence électronique

Françoise Rétif, « Cette beauté qui tue. Le beau et le mythe des sirènes », Germanica [En ligne], 37 |

2005, mis en ligne le 07 janvier 2010, consulté le 06 octobre 2020. URL : http://

journals.openedition.org/germanica/453 ; DOI : https://doi.org/10.4000/germanica.453

Ce document a été généré automatiquement le 6 octobre 2020.

(c) Tous droits réservés 


\title{
Cette beauté qui tue. Le beau et le mythe des sirènes
}

\author{
Françoise Rétif
}

\section{Le chant des sirènes}

1 À l'origine, les sirènes ne sont pas belles. C'est leur chant qui est beau; et c'est leur chant qui est fatal. Circée avertit Ulysse : gare à leur voix! De leur être physique, dans l'Odyssée, il n'est pas question. On suppose qu'elles étaient plus aériennes que marines, sans doute mi-femmes mi-oiseaux, puisque certaines versions tardives de la légende affirment qu'après le passage d'Ulysse elles se seraient précipitées du haut du rocher où elles séjournaient - certaines représentations les montrent en effet, très laides, difformes, les griffes de leurs pattes d'oiseaux rivées au rocher.

On sait comment, au sortir du royaume d'Hadès et sur les conseils de Circée, Ulysse échappa par la ruse aux sirènes en bouchant avec de la cire les oreilles de ses compagnons et en se faisant attacher au mât de son bateau. Car on n'entend pas impunément le beau chant des sirènes : " celui qui les approche sans être averti et les entend, jamais sa femme et ses petits enfants ne se réunissent près de lui et ne fêtent son retour ", prévient Circée. C'est Ulysse lui-même, échoué au pays des Phéaciens, qui fait le récit de la façon dont il réussit à triompher du danger. Si donc c'est en auditeur passif, ainsi que le soulignent Adorno et Horkheimer dans Die Dialektik der Aufklärung, qu'il subit le chant des sirènes, c'est ensuite en poète et conteur, succédant au divin Démodocos, qu'il en fait le récit. "Entendre le chant des sirènes, c'est d'Ulysse qu'on était devenir Homère ", note Maurice Blanchot dans Le Livre à venir. C'est parce qu'il a subi l'épreuve du chant des sirènes et l'a surmontée qu'Ulysse est devenu aède. En sachant attacher son corps, en s'imposant des liens et des règles - peut-on aller jusqu'à dire : le carcan d'une forme - il surmonte l'épreuve qui le ramène en Ithaque. Les sirènes se trouvent au cœur du voyage initiatique qui mène Ulysse de la magicienne Circée qui tisse en chantant à sa femme Pénélope qui tisse inlassablement sans jamais chanter. 
3 «Le chant des sirènes, écrit Tzvetan Todorov dans Poétique de la prose, est cette poésie qui doit disparaître pour qu'il y ait vie, et cette réalité qui doit mourir pour que naisse la littérature. Le chant des sirènes doit s'arrêter pour qu'un chant sur les sirènes puisse apparaître. Si Ulysse n'avait pas échappé aux sirènes, s'il avait péri à côté de leur rocher, nous n'aurions pas connu leur chant; tous ceux qui l'avaient entendu, en étaient morts et ne pouvaient pas le retransmettre. Ulysse, en privant les sirènes de vie, leur a donné, par l'intermédiaire d'Homère, l'immortalité »1. Todorov touche ici au cœur du problème : il s'agit bien du passage d'une forme de chant à une autre- (ce qu'il désigne comme le passage de la poésie à la littérature ${ }^{2}$ ), de la représentation symbolique d'un moment crucial dans l'histoire de l'art et de l'esthétique. Mais l'on ne peut se départir du sentiment que l'éminent critique se résout rapidement au sort qui est imparti aux sirènes dans le mythe, sans peut-être aller jusqu'au bout de la question de savoir pourquoi ce chant si beau et celles qui le produisent doivent être sacrifiés. Qu'est-ce qui rend ce sacrifice nécessaire ? Qu'est-ce que ce «beau » qui doit, nous diton, cesser de se faire entendre pour qu'il y ait «vie »? Pourquoi est-il justement représenté par des sirènes, ces femmes à mi-chemin entre animalité ou nature et humanité ou culture?

L'on sait que Adorno et Horkheimer situent Ulysse à l'origine de l'histoire de la raison ; la rencontre d'Ulysse et des sirènes nous raconte selon eux le début de la soumission de la nature. Après le passage d'Ulysse, le charme des sirènes est neutralisé ; elles qui étaient l'incarnation même de la poésie, du chant, deviennent objets du récit, de l'art. Mais on peut voir dans cette lutte à mort entre Ulysse et les sirènes, je le disais, le début d'une autre conception de la poésie et de l'art. Ce sont deux conceptions du chant qui s'opposent ici. Le chant des sirènes, aussi beau soit-il, ne "traite (que) de lui-même » . Ou peut-être est-il si beau parce qu'il ne traite que de lui-même ? C'est en tout cas ce qu'affirme Todorov : «La parole la plus belle est celle qui se parle »4. On le sait : beauté, mort et narcissisme sont souvent associés et incarnés dans des personnages féminins ou féminisés. Le chant d'Ulysse est orienté sur la ligne du temps, il a un objectif: obtenir de l'aide des Phéaciens; il a un but: le retour. Celui des sirènes, au contraire, n'a d'autre but que de mettre fin à l'action. Signe annonciateur: le vent tombe à l'approche de l'île des sirènes. Leur chant enlise/s'enlise dans la contemplation infinie du passé, de la matière à l'abandon, de la mort. On a ainsi, d'un côté, le chant naturel, informe, expression du désœuvrement réflexif, narcissique, celui des sirènes; de l'autre, celui qui est mis en forme, sans jamais perdre de vue son but, expression de la maitrise, celui d'Ulysse. L'un répond aux exigences de la « raison instrumentale », pour reprendre l'expression des philosophes de l'école de Francfort蕰; l'autre est résolument incantatoire, magique, lyrique. Peut-être est-ce là le début d'une opposition entre genre épique et genre lyrique. En tout cas, l'opposition existe moins entre poésie et vie qu'entre un certain type ou genre de poésie et un certain type ou genre de vie. Elle s'incarne de plus, ne l'oublions pas, dans l'opposition des sexes - ou genres. Le « beau sexe », ici, est plus que jamais associé à la " nature ». Le beau sexe relève de la nature, et donc l'art, le chant, le beau qu'il incarne aussi. C'est peut-être là la raison essentielle de l'exclusion (ou de la mort) prétendument nécessaire des sirènes : elles incarnent la nature comme source de beauté ou la beauté liée au sensible, au corps et à sa contingence, à l'expression immédiate et intérieure de la vie. L'exclusion des sirènes relève d'un choix esthétique, voire du choix d'une esthétique. "Si l'on s'arrête à la seule beauté corporelle et sensible, on prend le risque de se perdre dans le domaine de la contingence et l'on oublie les réalités authentiques ", note Platon dans Le Banquet. 


\section{La beauté des sirènes}

L'ère chrétienne fait subir aux sirènes, au cours des nombreux siècles durant lesquels elles mènent une vie plus ou moins souterraine, un certain nombre de mutations : elles troquent leurs pattes et ailes d'oiseaux pour une queue de poisson. Elles sont passées sous la surface de l'eau, dans l'élément où l'homme justement ne peut que mourir. Car l'homme est désormais solidement ancré sur terre : il a quitté sa frêle embarcation, soumise aux caprices de l'élément aquatique, pour chevaucher en chevalier la nature domptée, apprivoisée, et donc anoblie, son fidèle destrier. La surface de l'eau est devenue frontière. Le désir de rencontre, venant de l'homme, ne peut qu'être mortel. Si c'est la sirène qui surgit, elle est presque automatiquement déstabilisatrice : car elle est l'autre absolu, venu d'un autre monde, incompatible. Mais surtout la sirène (et ses cousines germaines, les nymphes, lorelei et ondines) est devenue résolument belle. Elle est certes toujours fatale. Mais la beauté fatale est désormais une beauté plastique. Dépossédée de son chant, la sirène est devenue objet de contemplation. Quand, au début $\mathrm{du}$ XIX $\mathrm{e}^{\mathrm{e}}$ siècle, la figure de la sirène ressurgit subitement et massivement dans l'espace germanique, le son a depuis longtemps perdu de l'importance par rapport à la vision, l'écriture a écarté la tradition orale, le texte et l'œil l'emportent sur la musique et l'oreille. La sirène est celle qui avant tout autre chose, nous allons le constater, séduit l'œil. Et c'est souvent le regard, plus que le chant, qui est son arme.

6 La Lorelei (ou Lore Lay), née, au tout début du XIX (1801-1802), de l'imagination nourrie de littérature antique de Clemens Brentano, est une jeune fille allemande d'un charme indicible, à la fois femme fragile et femme fatale. Elle ne chante pas; ce sont ses yeux, « deux flammes » qui enivrent et ensorcellent, incarnant le mystère de la séduction magique, diabolique. Réécrivant, un siècle plus tard, la ballade de Brentano, Guillaume Apollinaire ne s'y trompe pas et le souligne avec complaisance dès le début de sa traduction libre :

ô belle Loreley aux yeux pleins de pierreries

De quel magicien tiens-tu ta sorcellerie

Je suis lasse de vivre et mes yeux sont maudits

Ceux qui m'ont regardée évêque en ont péri

Mes yeux ce sont des flammes et non des pierreries

Jetez jetez aux flammes cette sorcellerie [...].

7 L'ondine de Friedrich de la Motte Fouqué (1811) est elle aussi décrite dans tout l'éclat de sa beauté, "une merveilleuse blondinette» aux "traits enchanteurs" que le chevalier cherche à graver à jamais en son âme. Cependant cette beauté capricieuse et magique de sauvageonne ne s'accomplit et ne prend toute son ampleur qu'après s'être transformée, du fait du mariage et donc de la possibilité d'acquérir une âme, en une beauté sage, douce, angélique - celle de la parfaite épouse chrétienne. La vraie beauté, "la splendeur du vrai », chez Fouqué, est celle de l'union impossible de la nature et de la civilisation christianisée, innocente et libérée de tout péché. La « vraie » beauté sert la "construction d'un ordre apparemment naturel des sexes $\|^{5}$ au sein de la société bourgeoise capitaliste en train de se structurer aux alentours de 1800.

Le chant d'Ondine, au onzième chapitre du conte, dit la vérité de l'origine (celle de Berthalda) ; il sert avant tout à nouer et dénouer l'action, il est un élément de la chaîne 
narrative, mais il n'a pas de valeur lyrique. Il est vrai, mais il n'est pas beau. Le chant d'Ondine n'a plus de valeur esthétique en soi. Un peu plus tard, exilée volontaire enfermée à l'abri des murailles du château de Ringstetten, la jeune fille de naguère, légère et capricieuse, élémentaire et incontrôlable, devient l'incarnation de l'irrationnel apprivoisé, domestiqué, christianisé, maîtrisé, le temps d'un récit. À la fin, chacun a repris sa place, la séparation a repris ses droits. Le récit, c'est-à-dire la réécriture du mythe, ne fait rien d'autre que de refléter le processus de création: l'irruption et la capture momentanée de l'irrationnel, merveilleux ou fantastique, dans la forme close $d u$ conte $^{6}$. La sirène est la métonymie d'une forme, d'un genre (littéraire). Et le genre (sexuel) est métonymique d'un genre (littéraire).

9 La Lorelei de Heinrich Heine, en 1822, allie le chant de «l'antique syrène » et la beauté de la magicienne de Brentano. Son chant est « merveilleux » et "puissant »; cependant c'est bien sur l'élément visuel, sur la description de la représentation, qu'insiste Heine, sur la beauté resplendissante, apollinienne de la sirène :

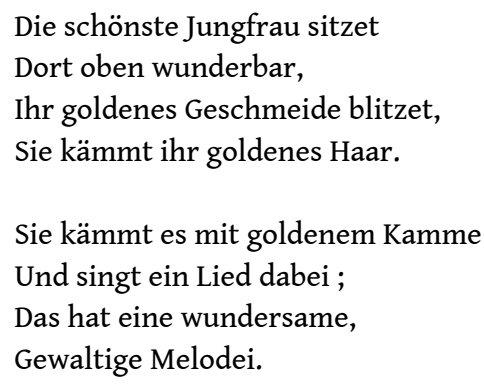

Le chant de la Lorelei est devenu image, représentation, figuration, Bild. La sirène, la Lorelei est devenue muse ${ }^{7}$, imagination, Einbildungs-kraft. Elle est image et force qui suggère les images. Son chant propre est diabolisé, rejeté (« und das hat mit ihrem Singen/Die Lorelei getan »), après avoir été qualifié, objectivé. Mais l'évocation au cœur du poème, emprisonnée dans la forme du poème, à l'intérieur des remparts des stophes-cadre, n'aboutit jamais au chant lui-même. Le chant n'est jamais qu'évoqué par le poète; le chant de la sirène, on ne l'entend pas. Ce qu'on entend est le chant du poète, qui fait de la représentation de la Lorelei un chant.

C'est déjà ce que suggérait la dernière strophe de la ballade de Clemens Brentano :

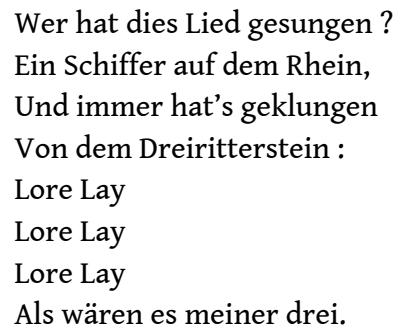

De la Lorelei, il ne reste que l'écho, non de sa voix, mais de son nom, prononcé par celui qui chante le chant du poète. C'est seulement ainsi, presque entièrement dématérialisé, désincarné par le jeu des échos successifs et de l'éloignement, que le son peut être, timidement, peureusement, évoqué. La beauté magique du son ne peut être évoquée qu'au moment de la perte, qu'en souscrivant à sa perte. Il semble que la beauté plastique serve à faire écran à l'autre beauté, celle du chant, dont il ne reste que l'écho dans le nom. Telle Eurydice, Lore Lay est ce à quoi le poète pense devoir renoncer, ce qu'il croit devoir sacrifier pour que le poème advienne. Dans ce travail d'auto-censure ou d'autocastration, "la Raison se saisirait de l'art pour en faire la technique de son auto- 
représentation $»^{8}$. Et l'œil serait l'organe de ce processus, de cette prise de distance par rapport au monde sensible, à la nature, l'organe de cette abstraction grandissante 9 .

On constate une insistance encore plus marquée, et comme redoublée, à décrire la beauté plastique, la magnificence des atours et vêtements, chez Gérard de Nerval, dans Lorely, Souvenirs d'Allemagne. Nous sommes en 1852, l'instrumentalisation presque déjà médiatique (on n'est pas loin de l'icône publicitaire!) de la beauté féminine est en marche :

Vous la connaissez comme moi, mon ami, cette Loreley ou Lorelei, - la fée du Rhin, dont les pieds rosés s'appuient sans glisser sur les rochers humides de Bacharach, près de Coblenz. Vous l'avez aperçue sans doute avec sa tête au col flexible qui se dresse sur son corps penché. Sa coiffe de velours grenat, à retroussis de drap d'or, brille au loin comme la crête sanglante du vieux dragon de l'Eden.

Sa longue chevelure blonde tombe à sa droite sur ses blanches épaules, comme un fleuve d'or qui s'épancherait dans les eaux verdâtres du fleuve. Son genou plié relève l'envers chamarré de sa robe de brocart, et ne laisse paraître que certains plis obscurs de l'étoffe verte qui se colle à ses flancs.

Son bras gauche entoure négligemment la mandore des vieux Minnesaenger de Thuringe, et entre ses beaux seins aimantés de rose, étincelle le ruban pailleté qui retient faiblement les plis de lin de sa tunique. Son sourire est doué d'une grâce invincible et sa bouche entrouverte laisse échapper les chants de l'antique syrène. Je l'avais aperçue déjà dans la nuit, sur cette rive où la vigne verdoie et jaunit tour à tour, relevée au loin par la sombre couleur des sapins et par la pierre rouge de ces châteaux et de ces forts [...]. Eh bien, mon ami, cette fée radieuse des brouillards, cette ondine fatale, comme toutes les nixes du Nord qu'a chantées Henri Heine, elle me fait signe toujours : elle m'attire encore une fois ! Je devrais me méfier pourtant de sa grâce trompeuse, - car son nom même signifie en même temps charme et mensonge ; et une fois déjà je me suis trouvé jeté sur la rive, brisé dans mes espoirs et dans mes amours, et bien tristement réveillé d'un songe heureux qui promettait d'être éternel.

14 Tout est dit : la sirène, d'abord dépossédée de son chant, c'est-à-dire de son savoir et de son art, puis réduite à l'écho de son nom, ne signifie ici plus que "charme et mensonge » - l'éternel féminin.

Plus le $\mathrm{XIX}^{\mathrm{e}}$ siècle progresse, plus la sirène est maltraitée. Il faudrait évoquer le si curieux fragment d'Achim von Arnim, dit Fragment de Mélusine $e^{10}$, dans lequel le petit garçon, sur l'insistance de son père, accepte de se faire enlever les nageoires qu'il porte dans le dos, résidu suranné de temps anciens, du règne de la grande Mère fondatrice, de la force créatrice de la nature. La petite sirène d'Andersen, quant à elle, renonce non seulement à sa queue de poisson, mais aussi à sa langue; pour avoir le droit d'approcher le beau prince dont elle s'est éprise, il lui faut correspondre au canon culturel de beauté. La sirène doit renoncer doublement à elle-même, à son chant et à son type de beauté. Sois belle et tais-toi! Mais sois belle selon les formes qui te seront dictées.

16 Dans la célèbre nouvelle de Kafka (1917), les sirènes, " plus belles que jamais », ont fait de leur silence la plus redoutable des armes. On a changé de siècle, et probablement aussi d'esthétique: "le chant énigmatique (des sirènes) est (désormais) puissant par son défaut ", ainsi que l'affirmera Maurice Blanchot dans Le Livre à venir.

17 Cependant, s'il est avéré que le chant de la sirène s'est bel et bien arrêté, comme le remarquait avec fatalité Todorov - il serait sans doute plus exact de dire d'ailleurs qu'il a été arrêté - ; si la sirène, devenue objet ambigu de contemplation, est dépossédée de son chant, il ne fait pas de doute que sa réapparition même, son resurgissement 
brusque et massif au début du XIX $x^{e}$, et même dès la fin du XVIII ${ }^{e}$ siècle, marque - avec certes toutes les réserves et limites que je viens d'évoquer et qui ne font peut-être que souligner la force du danger en présence, c'est-à-dire la puissance du resurgissement une réhabilitation, ou la tentative de réhabilitation d'une conception naturelle, sensible, dionysiaque, spontanée, en deça des formes du Beau, ainsi que d'une esthétique qui redonne au son, au chant, à la musique toute sa place - voire en fait « le plus haut de tous les arts » (Friedrich Schlegel).

Ce n'est pas un hasard si E.T.A Hoffmann mit en musique, fit un opéra de la nouvelle de Fouqué. Sa cousine slave, la Roussalka de Pouchkine, créée à peu près à la même époque, appellera elle aussi une mise en forme musicale (par Henri Duparc en 1897, resté à l'état de fragment, et par Dvorak en 1901). Ce n'est pas un hasard si, dans un conte populaire, forme de " poésie naturelle » (Naturpoesie), Die Nixe am Teich des frères Grimm, l'on voit la sirène réclamer son dû, les attributs de son art qui lui ont été dérobés, en échange de l'amant enlevé par ses soins : la flûte, le peigne et le métier à tisser. Quand on sait que les mots grecs et latins qui désignaient le peigne, à la fois comme instrument pour lisser la chevelure et pour peigner les fibres textiles, faisaient référence également aux poils pubiens, l'on comprend que ce que réclame la nymphe du lac symbolise les exercices différents de son pouvoir et de son art. Le conte populaire (Volksmärchen) (souvent raconté par des femmes à ces collecteurs-écrivains que furent les frères Grimm) rend à la sirène ce qui lui appartenait jadis, là où le conte littéraire (Kunstmärchen) cherchait encore à se l'approprier ou à le neutraliser.

À l'origine de ce renouveau de la figure de la nymphe (ou sirène ou ondine etc.), il faut sans doute, une fois de plus, placer Gœthe. Car dès 1779, il n'hésite pas à faire surgir de l'eau une «femme humide»- humide est le seul qualificatif utilisé pour la désigner qui par son chant et par son chant uniquement - de sa beauté, il n'est pas fait état attire le pêcheur vers le fond de la "rosée éternelle » en lui vantant la beauté du monde. Si la sirène de Gœthe loue ce qui n'est peut-être rien d'autre que les chimères d'un bonheur impossible, voire fatal, le poète ose quant à lui redonner voix à la sirène et il ose en faire le porte-parole d'une beauté qui se regarde moins qu'elle ne se donne à jouir.

20 Ainsi voit-on dès la fin du xvIII ${ }^{\mathrm{e}}$ siècle, au moment où l'esthétique se constitue en discipline, apparaître la sirène au cœur d'un débat esthétique qui va s'amplifier au cours du XIXe.

21 Il faudra ensuite attendre longtemps, le milieu du $x^{e}$ siècle, pour entendre pour la première fois dans la littérature occidentale une sirène, une ondine se mettre ellemême en scène à la première personne et, libre et autonome, par son chant qui dépasse les frontières des genres, de tous les genres ${ }^{11}$, incarner l'art, une création au féminin qui ne se dissocie pas de l'art d'aimer ni de l'amour de vivre. Mais de beauté, il n'est nullement question dans Undine geht d'Ingeborg Bachmann (1961). 


\section{NOTES}

1. Poétique de la prose, Éditions du Seuil, 1971, p. 71.

2. Mais on sait que le mot « littérature », dans l'espace germanique au moins, ne fut employé qu'à partir du XVIII ${ }^{\mathrm{e}}$ siècle.

3. Poétique de la prose, op. cit., p. 71.

4. Idem.

5. Claudia Öhlschläger, Unsägliche Lust des Schauens. Die Konstruktion der Geschlechter im voyeuristischen Text, Freiburg im Breisgau, Rombach Verlag, 1996, p. 28.

6. Cf. à ce propos: Françoise Rétif, Le Genre et la sirène, in : Michèle Guéret-Laferté, Daniel Mortier, D'un genre à l'autre, PUR, à paraître.

7. Cf. à ce sujet les articles de Geneviève Fraisse dans : La Controverse des sexes, PUF, Quadrige, 2001.

8. Jean-Luc Nancy, Une pensée finie, Galilée, p. 157.

9. Cf. Harmut Böhme, "Sinne und Blicke.Zur mythopoetischen Konstitution des Subjekts ", In : Hartmut Böhme, Natur und Subjekt, Frankfurt a.M. 1988, p. 223 : « denn es geht nicht allein [...] um die Emanzipation vom unbändigen Trieb durch Aufbau einer rationalen Kontrollinstanz, sondern zugleich um einen Abstraktions- und Idealisierungsprozeß [...], der libidoökonomisch als Transformation objektgerichteter Triebenergie in narzißtische Idealisierungen zu verstehen ist. Dieser Vorgang spiegelt sich im Aufstieg des Auges. »

10. Cf. Achim von Arnim, Mélusine. Contes et récits, édition, préface et traduction de Françoise Rétif, Paris, Corti, 1996.

11. Genres littéraires et genres sexuels, Cf. à ce propos : Françoise Rétif, « Quatre mythes de la création", in: Françoise Rétif et Ortrun Niethammer, Mythos und Geschlecht/Mythes et différences des sexes, Heidelberg, Winter Verlag, Septembre 2005, et F.R. : « Konstruktion und Dekonstruktion der Geschlechterdifferenzen als Kulturkonflikte", (version abrégée) in : Akten des XI. Internationalen Germanisten-kongresses Paris 2005, Jahrbuch für Internationale

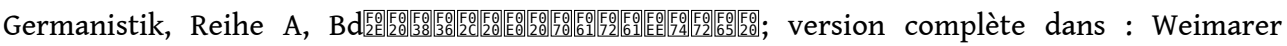
Beiträge, 2006.

\section{RÉSUMÉS}

L'ère chrétienne fait subir aux sirènes, au cours des nombreux siècles durant lesquels elles mènent une vie plus ou moins souterraine, un certain nombre de mutations : elles troquent leurs pattes et ailes d'oiseaux pour une queue de poisson. Elles sont passées sous la surface de l'eau, dans l'élément où l'homme justement ne peut que mourir. Car l'homme est désormais solidement ancré sur terre : il a quitté sa frêle embarcation, soumise aux caprices de l'élément aquatique, pour chevaucher en chevalier la nature domptée, apprivoisée et donc anoblie, son fidèle destrier. La surface de l'eau est devenue frontière. Le désir de rencontre, venant de l'homme, ne peut qu'être mortel. Si c'est la sirène qui surgit, elle est presque automatiquement déstabilisatrice : car elle est l'autre absolu, venu d'un autre monde, incompatible. Mais surtout la sirène (et ses cousines germaines, les nymphes, lorelei et ondines) est devenue résolument belle. Elle est toujours fatale. Mais la beauté fatale est désormais une beauté plastique. Dépossédée de son 
chant, la sirène est devenue objet de contemplation. Quand, au début du XIX ${ }^{\mathrm{e}}$ siècle, la figure de la sirène ressurgit subitement et massivement dans l'espace germanique, le son a depuis longtemps perdu de l'importance par rapport à la vision, l'écriture a écarté la tradition orale, le texte et l'œil l'emportent sur la musique et l'oreille. La sirène est celle qui avant tout autre chose, nous allons le constater, séduit l'œil. Et c'est souvent le regard, plus que le chant, qui est son arme.

In der christlichen Ära erleben die Sirenen im Laufe der zahlreichen Jahrhunderte gewisse Wandlungen, wo sie ein mehr oder weniger unterirdisches Leben führen : ihre Vogelpfoten und Flügel werden durch einen Fischschwanz ersetzt. Sie sind unter die Wasserfläche untergetaucht, gerade ins für den Menschen tödlich wirkende Element. Nunmehr ist der Mensch in der Erde fest verankert : er hat sein schwaches, dem launenhaften Wasserelement geliefertes Boot verlassen, um als Wasserritter auf der gezahmten, bezwungenen, also veredelten Natur, seinem treuen Schlachtroß, zu reiten. Die Erdfläche ist ihm zur unüberbrückbaren Grenze geworden. Der dem Menschen entsprungene Wille zur Konfrontation kann nur tödlich wirken. Taucht die Sirene auf, so wirkt sie quasi unmittelbar zerstörerisch : denn sie ist das andere schlechthin, das von einer anderen, unversöhnlichen Welt herstammt. Vor allem ist aber ist die Sirene (und deren Kusinen die Nymphen, Loreleien und Undinen) entschlossen schön geworden. Sie wirkt immer fatal. Doch erweist sich nunmehr die fatale Schönheit als plastische Schönheit. Die ihres Gesangs beraubte Sirene ist zum Gegenstand der reinen Anschauung geworden. Als zu Beginn des 19. Jahrhunderts die Sirenenfigur unvermittelt und massiv im deutschsprachigen Raum wiederauftaucht, hat das Gehör dem Gesicht gegenüber schon lange an Bedeutung verloren; die Schrift hat die mündliche Überlieferung vertrieben, Text und Auge gewinnen nun die Oberhand über Musik und Ohr. Die Sirene ist diejenige, die vor allem das Auge entzückt, wie es hier gezeigt werden soll. Und oft greift sie mehr zur Waffe des Blicks als des Gesangs.

\section{INDEX}

Mots-clés : beauté, laideur

\section{AUTEUR}

\section{FRANÇOISE RÉTIF}

Université de Rouen/CR2A 